\title{
El Laboratorio del Patrimonio del CSIC diseña REDAR, una red al servicio de la Arqueología
}

\begin{abstract}
El Consejo Superior de Investigaciones Científicas ha realizado, a través del Laboratorio de Patrimonio (IEGPS), la "Primera encuesta nacional dirigida a empresas de la arqueología comercial". Este estudio pionero recopila información social y económica sobre la actividad y con los resultados se ha elaborado la primera base de datos sobre la oferta de servicios de este mercado. Toda la información se volcará en un portal web de acceso abierto que estará disponible a principios de 2011.
\end{abstract}

La primera encuesta nacional a empresas de Arqueología fue realizada en 2009 por el Laboratorio de Patrimonio (IEGPS, CSIC), con la colaboración del Instituto de Estudios Sociales Avanzados (IESA, CSIC-Junta de Andalucía, Córdoba), el Instituto de Gestión de la Innovación y del Conocimiento (INGENIO-CSIC, Valencia) y el Centro de Ciencias Humanas y Sociales (CCHS-CSIC, Madrid).

La muestra estuvo constituida por 273 empresas y el índice de participación fue del 78\%. Se realizó entre junio y noviembre de 2009 a través de un sistema de encuestación telefónico y asistido por una aplicación en línea. Según se recoge en esta base de datos, Andalucía, Cataluña y Madrid son las comunidades autónomas con más empresas dedicadas a la Arqueología (45, 44 y 38 , respectivamente).

\section{El portal web de acceso abierto pretende centralizar información sobre el sector arqueológico español}

Los resultados del cuestionario permiten trazar, por primera vez, el perfil de las empresas españolas que centran su actividad en temas arqueológicos: periodo temporal en el que surgen; forma juridica; actividades; principales clientes; volumen de facturación; personal... mientras que el análisis estadístico de los datos permite elaborar requerimientos del sector en materia de innovación y transferencia de conocimiento.

Debido al éxito de esta actividad, y como consecuencia, en parte, de los resultados obtenidos, desde el Laboratorio de Patrimonio se está poniendo en marcha la construcción de un portal web, de acceso abierto, que pretende centralizar información sobre el sector arqueológico español.

Esta iniciativa tiene por objeto implementar una herramienta al servicio de los profesionales de la Arqueología, la comunidad científica y la ciudadanía, que proporcione y recopile información sobre la evolución y necesidades del sector. Para ello, el portal web se nutriria de información proveniente de trabajos ya desarrollados, pero también de datos recogidos a través del propio portal, permitiendo a los usuarios la actualización de determinados contenidos y la cumplimentación de formularios y encuestas.

Este portal web se materializará a finales de 2010, y en un primer momento constará de los siguientes contenidos: la primera base de datos en España sobre la oferta de actividad existente en materia de gestión patrimonial, identificando los principales agentes e instituciones vinculados al sector arqueológico. Con ello se pretende visibilizar, por primera vez, información sobre la estructura y evolución de este mercado, localizar la oferta y demanda existente en España en torno a esta actividad, de modo que opere como base de datos de la Arqueología profesional en España para incentivar la mejora de la competitividad empresarial en el sector específico del patrimonio cultural. Pero, además, el portal busca generar nueva información, a través de su propio desarrollo y actualización, propiciando la sistematización y el análisis periódico de los datos y su materialización en documentos de acceso general. Finalmente, se incluye una base de datos sobre legislación vinculada a la gestión y protección del patrimonio cultural en España.

Cabe señalar que esta iniciativa se constituye a través de un portal abierto que pone la información, de una forma sistematizada, al servicio de esta comunidad, pero también servirá para detectar necesidades que posteriormente puedan ser satisfechas a través de la implementación de nuevas herramientas. Para ello, el Laboratorio de Patrimonio ha contactado con todas las empresas que participaron en el estudio, para informarles de esta actividad y recopilar información sobre el tipo de funcionalidades que estos agentes demandarian a través de esta iniciativa, y así hacerles participes del diseño del prototipo del portal web y de su posterior funcionamiento, garantizando su utilidad. Además, y una vez implementado, se pretende la búsqueda y localización de más agentes e instituciones del sector, a fin de que puedan incorporarse a la iniciativa. Para ello se ha puesto al servicio de esta comunidad el siguiente correo electrónico: redar@iegps.csic.es

En definitiva, a través de esta actividad se ejerce una acción cognitiva pionera en España y en Europa, un paso necesario para vertebrar, organizar, centralizar y dar salida y acceso abierto al conocimiento resultado de la investigación desarrollada por los distintos agentes implicados, al mismo tiempo que se concibe y diseña como vehículo para la transferencia de conocimiento en el sector.

\section{Eva Parga Dans}

Laboratorio de Patrimonio (IEGPS-CSIC) 